\title{
Effect of Non-Uniform Basic Temperature Gradient on the Onset of Rayleigh-Bénard-Marangoni Electro-Convection in a Micropolar Fluid
}

\author{
Thadathil Varghese Joseph ${ }^{1}$, Sree Ramaiah Manjunath ${ }^{2}$, Subbarama Pranesh ${ }^{1}$ \\ Department of Mathematics, Christ University, Bangalore, India \\ ${ }^{2}$ Department of Mathematics, BNMIT, Bangalore, India \\ Email: drmanjus@gmail.com,pranesh.s@christuniversity.in,joseph.tv@christuniversity.in
}

Received April 24, 2013; revised May 24, 2013; accepted June 2, 2013

Copyright (C) 2013 Thadathil Varghese Joseph et al. This is an open access article distributed under the Creative Commons Attribution License, which permits unrestricted use, distribution, and reproduction in any medium, provided the original work is properly cited.

\begin{abstract}
The effects of electric field and non-uniform basic temperature gradient on the onset of Rayleigh-Bénard-Marangoni convection in a micropolar fluid are studied using the Galerkin technique. The eigenvalues are obtained for an upper free/adiabatic and lower rigid/isothermal boundaries. The microrotation is assumed to vanish at the boundaries. A linear stability analysis is performed. The influence of various micropolar fluid parameters and electric Rayleigh number on the onset of convection has been analysed. Six different non-uniform temperature profiles are considered and their comparative influence on onset is discussed.
\end{abstract}

Keywords: Rayleigh-Bénard-Marangoni; Non-Uniform Temperature Gradient; Electric Field; Micropolar Fluid; Galerkin Technique

\section{Introduction}

Convection due to variations of densities between two parallel plates caused by temperature difference and variations of temperature dependence surface tension in a horizontal fluid layer is of great importance because of its applications in science and engineering. Such convection in literature is known as Rayleigh-Bénard and Bénard-Maangoni convection. The developments of convection and corresponding heat transfer are examples of the physical phenomena to be encountered in these types of problems. The flow associated with Rayleigh-Bénard convection and Marangoni convection has been extensively reviewed in the literature. Particularly, Marangoni convection is found to be of importance in crystal growth melts and nucleate boiling. The problem of onset of convection driven by the combined effect of buoyancy and surface tension is also important in understanding many practical problems.

Pearson [1] was the first person to give a detailed mathematical analysis for the onset of convection driven by surface tension gradients. Nield [2] studied the problem of onset of convection driven by the combined effect of buoyancy and surface tension. Later many authors, Rudraiah [3], Maekawa and Tanasawa [4] studied the onset of convection in a horizontal layer of Newtonian fluid driven by both surface tension variations and buoyancy force by considering the non-deformable surfaces. The corresponding problem with deformable boundaries is studied by Sarma [5,6], Takashima [7], Wilson [8,9], Hashim and Wilson [10].

The application of strong electric field in a poorly conducting fluid can induce bulk motions. This phenomenon is known as Electro convection. In recent years, the study of the interaction of electromagnetic fields with fluid stated gain attention with the promise of new applications. Onset of natural convection in the presence of external electric field had been studied by Turnbull [11], Turnbull and Melcher [12], Takashima and Aldridge [13], Stiles [14], Stiles et al. [15], Siddheshwar [16], Siddheshwar and Abraham [17,18], Siddheshwar and Chan [19], Shivakumara et al. [20], Rudraiah et al. [21] and Siddheshwar and Radhakrishna [22].

Most of the previous studies were concerned with convection in Newtonian fluids. However, very less work has been done on convection in micropolar fluid with electric fields. The theory of micropolar fluids, developed by Eringen [23] is the best established theory of fluid with microstructure. Physically, the micropolar fluid consists of small, rigid, cylindrical suspended particles. Many classical flows were reexamined using the micropolar fluid to study the effect of 
fluid microstructures. Thermal convection in micropolar fluids with uniform and non-uniform temperature gradients was studied by many authors (Datta and Sastry [24], Siddheshwar and Pranesh [25-27] Pranesh and Riya Baby [28], Pranesh and Arun Kumar [29]). The main result of all these studies is that the micropolar fluid heated from below is more stable compared to the fluid without suspended particles. To the best of our knowledge not much work is done in this field, except the above mentioned.

The main objective of this paper is to provide the theoretical framework for studying the thermo capillary instabilities in the presence of buoyancy instability in earth laboratories for electrically conducting micropolar fluid layers under an $\mathrm{AC}$ electric field and non-uniform temperature gradients. Eigen value of the problem is obtained using single term Galerkin method.

\section{Mathematical Formulation}

Consider an infinite horizontal layer of a Boussinesquian micropolar fluid layer of depth " $d$ ". The uniform electric field is directed along $z$-axis (see Figure 1). A Cartesian coordinate system is taken with the origin in the lower boundary and $z$-axis vertically up-wards. Let $\Delta T$ be the temperature difference between lower and upper boundaries of the fluid. The interface at the upper boundary has a temperature dependent surface tension $\sigma(T)$. Expanding $\sigma(T)$ by Taylor series about $T_{0}$, we get

$$
\sigma(T)=\sigma_{0}-\sigma_{1}\left(T-T_{0}\right)
$$

where, $\sigma_{1}=-\left(\frac{\mathrm{d} \sigma}{\mathrm{d} T}\right)_{T_{0}}$ and $\sigma_{0}=\sigma\left(T_{0}\right)$. The form of $\sigma(T)$ in Equation (1) was used by Pearson.

The governing equations for the problem are:

Equation of continuity:

$$
\nabla \cdot \boldsymbol{q}=0
$$

Conservation of Linear Momentum:

$$
\begin{aligned}
& \rho_{0}\left[\frac{\partial \boldsymbol{q}}{\partial t}+(\boldsymbol{q} \cdot \nabla) \boldsymbol{q}\right] \\
& =-\nabla p-\rho g \hat{k}+(2 \zeta+\eta) \nabla^{2} \boldsymbol{q}+\zeta \nabla \times \boldsymbol{\omega}+(\boldsymbol{P} \cdot \nabla) \boldsymbol{E},
\end{aligned}
$$

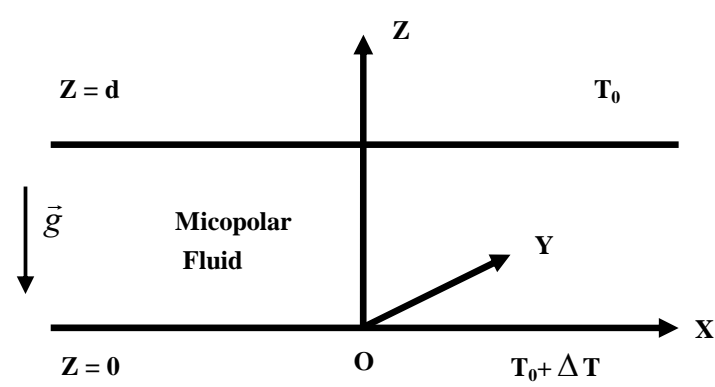

Figure 1. Schematic diagram of Rayleigh-Bénard situation for a fluid with suspended particles.

\section{Conservation of Angular Momentum:}

$$
\begin{aligned}
& \rho_{o} I\left[\frac{\partial \boldsymbol{\omega}}{\partial t}+(\boldsymbol{q} \cdot \nabla) \boldsymbol{\omega}\right] \\
& =\left(\lambda^{\prime}+\eta^{\prime}\right) \nabla(\nabla \cdot \boldsymbol{\omega})+\left(\eta^{\prime} \nabla^{2} \boldsymbol{\omega}\right)+\zeta(\nabla \times \boldsymbol{q}-2 \boldsymbol{\omega}),
\end{aligned}
$$

\section{Conservation of Energy:}

$$
\rho_{0} C_{V E}\left[\frac{\partial T}{\partial t}+\left(\boldsymbol{q}-\frac{\beta}{\rho_{o} C_{v}} \nabla \times \boldsymbol{\omega}\right) \cdot \nabla T\right]=K_{1} \nabla^{2} T,
$$

Equation of State:

$$
\rho=\rho_{o}\left[1-\alpha\left(T-T_{o}\right)\right] .
$$

Equation of State for Dielectric Constant:

$$
\varepsilon_{r}=\varepsilon_{r}^{0}-e\left(T-T_{0}\right),
$$

Faraday's Law:

$$
\nabla \times \boldsymbol{E}=0, \quad \boldsymbol{E}=-\nabla \varphi,
$$

Equation of Polarization Field:

$$
\nabla \cdot\left(\varepsilon_{o} \boldsymbol{E}+\boldsymbol{P}\right)=0, \boldsymbol{P}=\varepsilon_{o}\left(\varepsilon_{r}-1\right) \boldsymbol{E}
$$

where, $\boldsymbol{q}$ is the velocity, $\boldsymbol{\omega}$ is the spin, $p$ is the hydrodynamic pressure, $T$ is the temperature $\rho_{o}$ is the density of the fluid at a reference temperature $T=T_{0}$, $\zeta$ is the coupling viscosity coefficient, $\eta$ is the shear kinematic viscosity coefficient, $I$ is the moment of inertia, $\lambda^{\prime}$ and $\eta^{\prime}$ are bulk and shear spin viscosity coefficients, $\beta$ is a Micropolar heat conduction coefficient, $c_{v}$ is the specific heat, $\chi$ is the thermal conductivity, $\alpha$ is the coefficient of thermal expansion, $\boldsymbol{E}$ is the electric field, $\boldsymbol{P}$ is the dielectric polarization, $\boldsymbol{\varepsilon}_{o}$ is the electric permeability of free space and $\varepsilon_{r}$ is the dielectric constant.

The "Equations (2)-(9)" are solved subject to containment conditions appropriate for a rigid and thermally perfect conducting wall on the underside and a free surface on the upper side. This free surface is adjacent to a non-conducting medium and subject to a constant heat flux. Further, the no-spin boundary condition is assumed for micro-rotation. Since the shear stress for a non-classical fluid with suspended particles is no different from that of classical fluids, the boundary conditions for flat free boundaries used by Nield [2] in respect of Newtonian fluids are appropriate for micropolar fluids also.

In the quiescent state the velocity $\boldsymbol{q}$, the spin $\boldsymbol{\omega}$, the temperature $T$, the electric field $\boldsymbol{E}$, the pressure $p$, the density $\rho$, the dielectric polarization $\boldsymbol{P}$ have the following solution:

$$
\left.\begin{array}{l}
\boldsymbol{q}=0, \boldsymbol{\omega}=0, p=p_{b}(z), \boldsymbol{E}=E_{b}(z) \hat{k}, T=T_{b}(z), \\
\frac{-d}{\Delta T} \frac{\mathrm{d} T_{b}}{\mathrm{~d} z}=f(z)
\end{array}\right\}
$$


where $f(z)$ is a non-dimensional basic temperature gradient, satisfying the condition $\int_{0}^{1} f(z) \mathrm{d} x=1$.

Since the aim of the present study is to understand control of convection in a micropolar fluid layer by different basic temperature profiles, the various non-uniform basic temperature gradients considered in this study are represented in Table 1.

This type of basic temperature gradients arises due to sudden heating or cooling, radiation, through flow, etc.

In order to investigate the stability of the basic solution, infinitesimal disturbances are introduced in the form:

$$
\left.\begin{array}{l}
\boldsymbol{q}=\boldsymbol{q}_{b}+\boldsymbol{q}^{\prime}, \boldsymbol{\omega}=\boldsymbol{\omega}_{b}+\boldsymbol{\omega}^{\prime}, p=p_{b}+p^{\prime}, \boldsymbol{E}=\boldsymbol{E}_{b}+\left(E_{1}^{\prime}, E_{3}^{\prime}\right), \\
\rho=\rho_{b}+\rho^{\prime}, T=T_{b}+T^{\prime}, \boldsymbol{P}=\boldsymbol{P}_{b}+\left(P_{1}^{\prime}, P_{3}^{\prime}\right) .
\end{array}\right\}
$$

where the primes indicate that the quantities are infinitesimal perturbations assumed to be small and subscript ' $b$ ' indicates basic state value.

We assume the principal of exchange of stability to be valid and hence deal with only stationary convection. Introducing the electric potential $\phi^{\prime}$ and substituting "(11)" into "(2)-(9)" and using the basic state solution (10), we get linearized equation governing the infinitesimal perturbations in the form:

$$
\nabla \cdot \boldsymbol{q}^{\prime}=0
$$

$$
\begin{aligned}
\rho_{o}\left[\frac{\partial \boldsymbol{q}^{\prime}}{\partial t}\right]= & -\nabla p-\rho^{\prime} g \hat{k}+(2 \zeta+\eta) \nabla^{2} \boldsymbol{q}^{\prime}+\left(\zeta \nabla \times \boldsymbol{\omega}^{\prime}\right) \\
& +\left(\boldsymbol{P}_{b} \cdot \nabla\right) \boldsymbol{E}^{\prime}+(\boldsymbol{P} \cdot \nabla) \boldsymbol{E}_{b},
\end{aligned}
$$

$$
\begin{aligned}
& \rho_{o} I\left[\frac{\partial \boldsymbol{\omega}^{\prime}}{\partial t}\right] \\
& =\left(\lambda^{\prime}+\eta^{\prime}\right) \nabla\left(\nabla \boldsymbol{\omega}^{\prime}\right)+\left(\eta^{\prime} \nabla^{2} \boldsymbol{\omega}^{\prime}\right)+\zeta\left(\nabla \times \boldsymbol{q}^{\prime}-2 \boldsymbol{\omega}^{\prime}\right),
\end{aligned}
$$

$$
\begin{gathered}
\frac{\partial T^{\prime}}{\partial t}=\frac{\Delta T}{d}\left(\boldsymbol{q}^{\prime}-\frac{\beta}{\rho_{o} c_{v}} \nabla \times \boldsymbol{\omega}^{\prime}\right) f(z), \\
\rho^{\prime}=-\alpha \rho_{o} T^{\prime} .
\end{gathered}
$$

Table 1. Non-uniform basic temperature gradient profile.

\begin{tabular}{ccc}
\hline Model & Basic temperature gradients & $\boldsymbol{f ( z )}$ \\
\hline 1 & Linear & 1 \\
2 & Heating from below & $\left\{\begin{array}{cc}\varepsilon^{-1} & 0 \leq z<\varepsilon \\
0 & \varepsilon<z \leq 1\end{array}\right.$ \\
3 & Cooling from above & $\begin{cases}0 & 0 \leq z<1-\varepsilon \\
\varepsilon^{-1} & 1-\varepsilon<z \leq 1\end{cases}$ \\
5 & Step function & $\delta(z-\varepsilon)$ \\
6 & Inverted Parabolic & $2(1-z)$ \\
& Parabolic & $2 z$ \\
\hline
\end{tabular}

The perturbation "(12)-(15)" are non-dimensionalised using the following definitions:

$$
\left.\begin{array}{l}
\left(x^{*}, y^{*}, z^{*}\right)=\frac{(x, y, z)}{d}, W^{*}=\frac{W^{\prime}}{(\chi / d)}, \\
\boldsymbol{\omega}^{*}=\frac{\omega^{\prime}}{\left(\chi / d^{2}\right)}, t^{*}=\frac{t}{\left(d^{2} / \kappa\right)}, T^{*}=\frac{T^{\prime}}{\Delta T}, \\
\varphi^{*}=\frac{\varphi^{\prime}}{\left(\frac{e E_{0} \Delta T d}{1+\chi_{e}}\right)}, \Omega^{*}=\frac{\nabla \times \boldsymbol{w}}{\left(\chi / d^{3}\right)} .
\end{array}\right\}
$$

Using "(16)" in "(13)", operating curl twice on the resulting equation, operating curl on "(14)" and non-dimensionalising the two resulting equations and also “(15)", we get

$$
\begin{gathered}
R \nabla_{1}^{2} T+\left(1+N_{1}\right) \nabla^{4} W+N_{1} \nabla^{2} \Omega_{z} \\
+L \nabla_{1}^{2} T f(z)-L \frac{\partial}{\partial z}\left(\nabla_{1}^{2} \varphi\right) f(z)=0, \\
N_{3} \nabla^{2} \Omega_{z}-N_{1} \nabla^{\prime 2} W-2 N_{1} \Omega_{z}=0, \\
\nabla^{2} T+\left(W-N_{5} \Omega_{z}\right) f(z)=0, \\
\nabla^{2} \phi-\frac{\partial T}{\partial z}=0 .
\end{gathered}
$$

where the non-dimensional parameters $N_{1}, N_{3}, N_{5}, R$ and $L$ are given by:

$$
\begin{gathered}
N_{1}=\frac{\zeta}{\zeta+\eta} \text { (Coupling Parameter), } \\
N_{3}=\frac{\eta^{\prime}}{(\zeta+\eta) d^{2}} \text { (Couple Stress Parameter), }
\end{gathered}
$$

$N_{5}=\frac{\beta}{\rho_{o} C_{v} d^{2}}$ (Micropolar Heat Conduction Parameter),

$$
\begin{gathered}
R=\frac{\rho_{o} \alpha g \Delta T d^{3}}{\kappa(\zeta+\eta)} \text { (Rayleigh number), } \\
L=\frac{\varepsilon_{o} e^{2} E_{o}^{2} \Delta T}{\alpha_{1} g \rho_{o}\left(1+\chi_{e}\right) h} \text { (Electric Rayleigh number). }
\end{gathered}
$$

The infinitesimal perturbations $W, \Omega_{z}, T$ and $\phi$ are assumed to be periodic waves and hence these permits and normal mode solution in the following form:

$$
\left[\begin{array}{c}
W \\
\Omega_{z} \\
T \\
\phi
\end{array}\right]=\left[\begin{array}{c}
W(z) \mathrm{e}^{i\left(l_{x}+m_{y}\right)} \\
\Omega_{z}(z) \mathrm{e}^{i\left(l_{x}+m_{y}\right)} \\
T(z) \mathrm{e}^{i\left(l_{x}+m_{y}\right)} \\
\phi(z) \mathrm{e}^{i\left(l_{x}+m_{y}\right)}
\end{array}\right]
$$


where $l$ and $m$ are the horizontal components of the wave number $\boldsymbol{a}$.

We substituting “(22)” into "(18)-(21)”, then we get

$$
\begin{gathered}
\left(1+N_{1}\right)\left(D^{2}-a^{2}\right)^{2} W-R a^{2} T+N_{1}\left(D^{2}-a^{2}\right) G \\
-L a^{2} T f(z)+L a^{2} D \varphi f(z)=0, \\
N_{3}\left(D^{2}-a^{2}\right) G-2 N_{1} G-N_{1}\left(D^{2}-a^{2}\right) W=0, \\
\left(D^{2}-a^{2}\right) T+\left(W-N_{5} G\right) f(z)=0, \\
\left(D^{2}-a^{2}\right) \phi-D T=0,
\end{gathered}
$$

where $D=\frac{\mathrm{d}}{\mathrm{d} z}$ and $a^{2}=l^{2}-m^{2}$.

In "(23)-(26)", the asterisks have been omitted.

"(23)" and "(26)" are solved subject to the following boundary conditions:

$$
\left.\begin{array}{ll}
W=D W=T=G=0, & \text { at } z=0 . \\
W=D^{2} W+a^{2} M T=D T=G=0, & \text { at } z=1 .
\end{array}\right\}
$$

where $M$ is the Marangoni number.

"(27)" indicates the use of rigid, isothermal lower boundary and upper, free, thermally insulating boundary (with respect to perturbation). The condition on $G$ is spin-vanishing boundary condition.

We now use the single term Galerkin expansion technique to find the critical eigenvalue. Multiplying "(23)" by $W$, "(24)" by $G$, "(25)" by $T$ and "(26)" by $\phi$, integrating the resulting equations by parts with respect to $z$ from 0 to 1 , using the boundary conditions "(27)" and using $W=A W_{1}, G=B G_{1}, T=C T_{1}$ and $\phi=E \phi_{1}$ in which $A, B, C$ and $E$ are constants and $W_{1}, G_{1}, T_{1}$ and $\phi_{1}$ are trial functions, yield the following equation for the Marangoni number $M$ :

$$
M=\frac{\left[Y_{1} Y_{3}+R a^{2}\left\langle W_{1} T_{1}\right\rangle Y_{2}+L a^{2} Y_{4} Y_{2}-Y_{5}\right]}{a^{2} D W_{1}(1) T_{1}(1)\left(1+N_{1}\right) Y_{2}}
$$

where,

$$
\begin{aligned}
& Y_{1}=\left[\left(1+N_{1}\right)\left\langle T_{1}\left(D^{2}-a^{2}\right) T_{1}\right\rangle\right]\left[N_{3}\left\langle G_{1}\left(D^{2}-a^{2}\right) G_{1}\right\rangle-2 N_{1}\left\langle G_{1}^{2}\right\rangle\right], \\
& Y_{2}=N_{1} N_{5}\left\langle G_{1}\left(D^{2}-a^{2}\right) W_{1}\right\rangle\left\langle f(z) G_{1} T_{1}\right\rangle-\left\langle f(z) W_{1} T_{1}\right\rangle Y_{1}, \\
& Y_{3}=2 a^{2}\left\langle W_{1} D^{2} W_{1}\right\rangle-\left\langle\left(D^{2} W_{1}\right)^{2}\right\rangle-a^{4}\left\langle W_{1}\right\rangle, \\
& Y_{4}=\left\langle f(z) W_{1} T_{1}\right\rangle-\frac{\left\langle W_{1} D \varphi_{1} f(z)\right\rangle\left\langle\varphi_{1} D T_{1}\right\rangle}{\left\langle\varphi_{1}\left(D^{2}-a^{2}\right) \varphi_{1}\right\rangle}, \\
& Y_{5}=\frac{\left[N_{1}^{2}\left\langle T_{1}\left(D^{2}-a^{2}\right) T_{1}\right\rangle\left\langle\varphi_{1}\left(D^{2}-a^{2}\right) \varphi_{1}\right\rangle\left\langle W_{1}\left(D^{2}-a^{2}\right) G_{1}\right\rangle\left\langle G_{1}\left(D^{2}-a^{2}\right) W_{1}\right\rangle\right]}{\left\langle\varphi_{1}\left(D^{2}-a^{2}\right) \varphi_{1}\right\rangle} .
\end{aligned}
$$

In "(28)", $\langle--\rangle$ denotes integration with respect to $z$ between $z=0$ and $z=1$.

$M\left(W_{1}, G_{1}, T_{1}, \phi_{1}\right)$ in "(28)" is a functional and EulerLagrange equations for the extremisation of $M$ "(23)(26)".

The trial functions satisfying “(27)” are

$$
\left.\begin{array}{l}
W_{1}=z^{2}\left(1-z^{2}\right), \\
T_{1}=z(2-z), \\
G_{1}=z(1-z), \\
\phi_{1}=z^{2}\left(3 z-2 z^{2}\right) .
\end{array}\right\}
$$

such that they satisfy all the boundary conditions "(27)" except the one given by $D^{2} W+a^{2} M T=0$ at $z=1$, but the residual from this is included in the residual from the differential equations. Substituting "(29)" in "(28)" and performing the integration, we can calculate the critical Marangoni number $M_{c}$ which attains its minimum at $a_{c}^{2}$.

\section{Result and Discussion}

As per the motivation stated in the introduction to the paper we consider the effects of electric Rayleigh number, micropolar fluid parameters and non-uniform basic temperature distribution on the onset of Rayleigh-Bénard-Marangoni convection in a micropolar fluid layer. These three effects are respectively represented by the $L$, $\left(N_{1}, N_{3}\right.$ and $\left.N_{5}\right)$ and $f(z)$ in the Eigen value "(28)". Due to the thermodynamics restrictions the following values of $N_{1}, N_{3}$ and $N_{5}$ are chosen:

$$
0 \leq N_{1} \leq 1 ; \quad 0 \leq N_{3} \leq m ; \quad 0 \leq N_{5} \leq n
$$

where, $\mathrm{m}$ and $\mathrm{n}$ are finite, positive real numbers (see Siddheshwar and Pranesh [29]) and the values of $L$ are those chosen for Newtonian fluid problems. One linear and five non-linear basic temperature gradients are considered in the study (see Table 1). The results obtained in the study are depicted by the Figures 2-5. It is observ- 
ed that for the critical Marangoni number, $M_{c}$, the following inequality holds for the six models chosen in the study:

$$
M_{c_{4}}<M_{c_{3}}<M_{c_{6}}<M_{c_{2}}<M_{c_{1}}<M_{c_{5}}
$$

i.e., step function is the most destabilizing temperature profile and inverted parabolic temperature is the most stabilizing temperature profile. In the case of piecewise linear and step function profiles, the critical Marangoni number, $M_{c}$, depends on the thermal depth, $\varepsilon$, in addition to depending on the parameters of the problem. In the case of piecewise linear profile heating from below, cooling from above and step function profiles, the minimum value of $M_{c}$ is attained at $\varepsilon=0.95, \varepsilon=0.45$ and $\varepsilon=$ 0.75 respectively. With the above background and with the motive specified in the introduction we now discuss the results presented by the Figures 2-5.

Figure 2 is the plot of critical Marangoni number $M_{\mathrm{c}}$ versus coupling parameter $N_{1}$ for different non uniform temperature gradients and for different electric Rayleigh number $L$. Clearly $M_{c}$ increases with $N_{1}$. Increase in $N_{1}$ indicates increase in the concentration of micro elements. These elements consume the greater part of the energy of

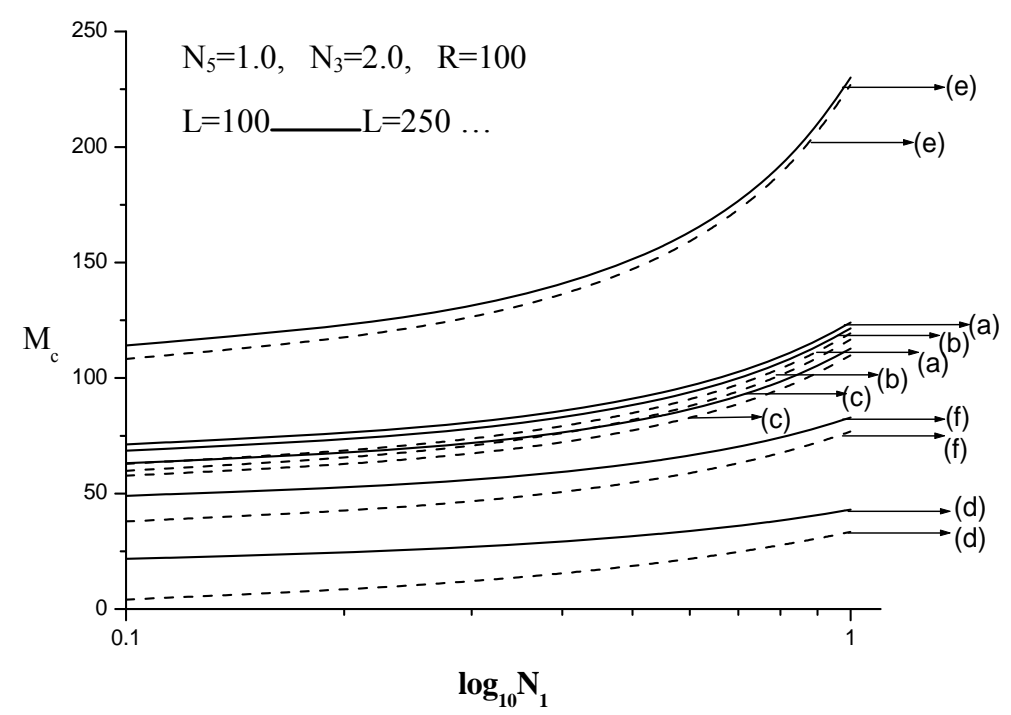

Figure 2. Plot of critical Maragoni number $M_{\mathrm{c}}$ versus coupling parameter $N_{1}$ for different non -uniform temperature gradient and different electric Rayleigh Number L. (a) Linear, (b) Heating from below, (c) Cooling from above, (d) Step function, (e) Parabolic, (f) Inverted parabolic.

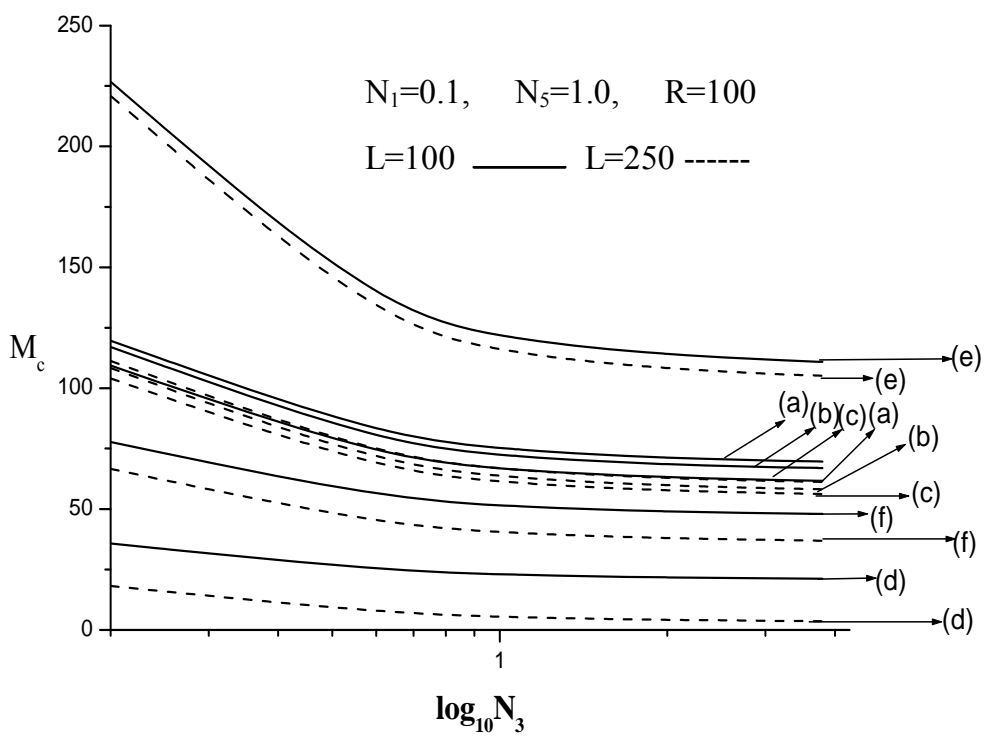

Figure 3. Plot of critical Maragoni number $M_{\mathrm{c}}$ versus coupling parameter $N_{3}$ for different non-uniform temperature gradient and different Electric Rayleigh number $L$. (a) Linear, (b) Heating from below, (c) Cooling from above, (d) Step function, (e) Parabolic, (f) Inverted parabolic. 


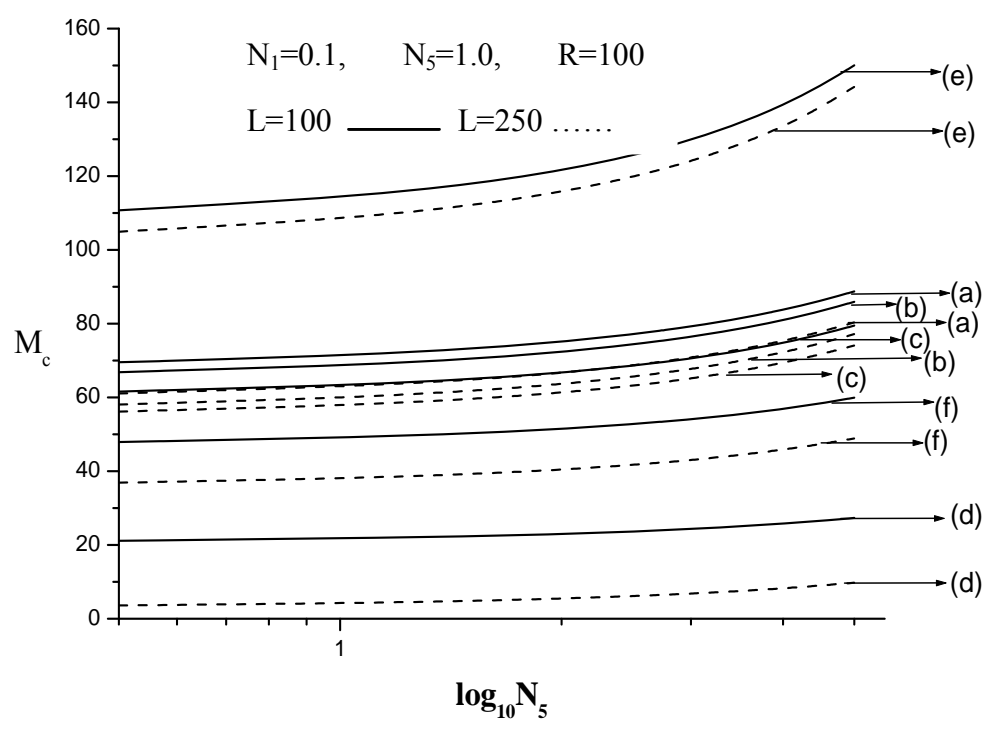

Figure 4. Plot of critical Maragoni number $M_{\mathrm{c}}$ versus coupling parameter $N_{5}$ for different non-uniform temperature gradient and different Electric Rayleigh number $L$. (a) Linear, (b) Heating from below, (c) Cooling from above, (d) Step function, (e) Parabolic, (f) Inverted parabolic.

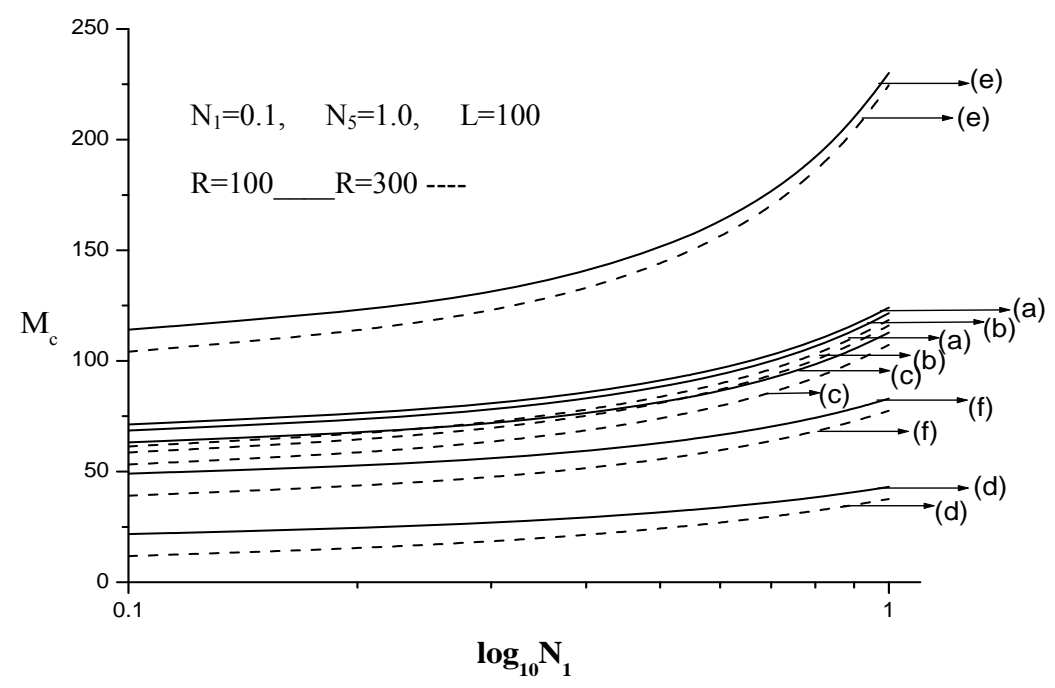

Figure 5. Plot of critical Maragoni number $M_{\mathrm{c}}$ versus coupling parameter $N_{1}$ for different non-uniform temperature gradient and different Rayleigh number R. (a) Linear, (b) Heating from below, (c) Cooling from above, (d) Step function, (e) Parabolic, (f) Inverted parabolic.

the system in developing the gyrational velocity of the fluid and as a result the onset of convection is delayed. Therefore, the increase $N_{1}$ is to stabilize the system. It is also observed that increase in electric Rayleigh number decreases the $M_{c}$. Electric Rayleigh number $L$ is the ratio of electric force to gravitational force. Thus, $L$ has a destabilizing effect on the system.

Figure 3 is the plot of $M_{c}$ versus coupling stress parameter $N_{3}$ for different non uniform temperature gradients and for different electric number $L$. Clearly $M_{c}$ decreases with increase in $N_{3}$. Increase in $N_{3}$, decreases the couple stress of the fluid which causes a decrease in mi- crorotation and hence makes the system more unstable.

Figure 4 is the plot of $M_{c}$ versus micropolar heat conduction parameter $N_{5}$ for different non uniform temperature profiles and for different electric number $L$. Clearly $M_{c}$ increases with $N_{5}$. When $N_{5}$ increases, the heat induced in the fluid due to these microelements also increases which reduces the heat transfer from bottom to top. The decrease in heat transfer is responsible for delaying the onset of instability. Thus increase in $N_{5}$ is to stabilize the system.

Figure 5 is the plot of $M_{c}$ versus coupling parameter $N_{1}$ for different non uniform temperature gradients and 
for different Rayleigh number $R$. Clearly $M_{c}$ increases with $N_{1}$. It is also observed that the increase in $R$ decreases the $M_{c}$ and ultimately we get the case of convection dominated by buoyancy force.

From the Table 2 it is clear that with an increase in $N_{1}$, $M_{c}$ becomes zero for higher values of $\mathrm{R}$ compared to Newtonian fluids. Similar results have been obtained in respect to the influences of $N_{3}$ and $N_{5}$. Treating Marangoni number as the critical parameter we find from the Table 3 that any particular infinitesimal disturbance can be destabilized with electric field. The results of the Table 3 are in respect of linear temperature gradient. This general observation on the destabilizing influence of electric field, however holds good for the other five temperature gradients as well. From Table $\mathbf{3}$ as well as Ta- ble 2, we also note that as $R$ increases $M_{c}$ decreases. From Table 4, it is found that the critical wave number is in general insensitive to the changes in the micropolar parameters but is influenced by the electric field. This indicates that, the convection cell at the onset increases in size with the increase in electric field. In the limit $L$ tends to zero, we recover the results of Siddheshwar and Pranesh [29] for Chandrasekhar number tends to zero from the present study.

The above result indicates that externally applied electric field is an effective means of controlling, RayleighBénard-Marangoni convection in mircopolar fluids. The result also suggests that Rayleigh-Bénard-Marangoni convection in Newtonian fluids may be delayed by adding micron sized suspended particles. Further by creating

Table 2. Critical Marangoni number $\left(M_{c}\right)_{j}(j=1$ to 6$)$ for different values of $L, R$ and $N_{1}, L=100, N_{3}=2.0, N_{5}=1.0$.

\begin{tabular}{|c|c|c|c|c|c|c|c|}
\hline$N_{1}$ & $\boldsymbol{R}$ & $M c_{1}$ & $M c_{2}$ & $M c_{3}$ & $M c_{4}$ & $M c_{5}$ & $M c_{6}$ \\
\hline \multirow[t]{11}{*}{0.5} & 0 & 94.4581 & 83.1598 & 69.7583 & 17.9915 & 148.788 & 66.3832 \\
\hline & 100 & 90.8073 & 79.509 & 75.8051 & 14.3407 & 145.137 & 62.7324 \\
\hline & 492.78 & 76.4677 & 65.1694 & 61.4655 & 0.001156 & 130.797 & 48.3928 \\
\hline & 500 & 76.2042 & 64.9058 & 61.2019 & -0.26243 & 130.534 & 48.1292 \\
\hline & 1000 & 57.9502 & 46.6518 & 42.9479 & & 112.28 & 29.8753 \\
\hline & 1818.29 & 28.0761 & 16.7778 & 13.0738 & & 82.4057 & 0.001178 \\
\hline & 2176.39 & 15.0026 & 3.70426 & 0.0003483 & & 69.3322 & \\
\hline & 2277.85 & 11.2985 & 0.000167 & & & 65.6281 & \\
\hline & 2500 & 3.18829 & & & & 57.5179 & \\
\hline & 2587.32 & 0.0004127 & & & & 54.33 & \\
\hline & 4075.47 & & & & & 0.0007194 & \\
\hline \multirow[t]{11}{*}{1.0} & 0 & 126.791 & 117.888 & 111.67 & 32.9602 & 228.56 & 85.6961 \\
\hline & 100 & 124.053 & 115.15 & 108.932 & 30.2221 & 225.822 & 82.958 \\
\hline & 500 & 113.101 & 104.197 & 97.9798 & 19.2698 & 214.87 & 72.0056 \\
\hline & 1000 & 99.4104 & 90.5069 & 84.2893 & 5.57929 & 201.179 & 58.3151 \\
\hline & 1203.75 & 93.8315 & 84.9281 & 78.7105 & 0.000422 & 195.601 & 52.7363 \\
\hline & 2500 & 58.3389 & 49.4355 & 43.2179 & & 160.108 & 17.2437 \\
\hline & 3129.69 & 41.0974 & 32.194 & 25.9764 & & 142.867 & 0.002182 \\
\hline & 4078.39 & 15.1211 & 6.21768 & 0.0000763 & & 116.89 & \\
\hline & 4305.47 & 8.90344 & 0.0000154 & & & 110.673 & \\
\hline & 4630.59 & 0.0013457 & & & & 101.77 & \\
\hline & 8347.39 & & & & & 0.0009143 & \\
\hline
\end{tabular}


Table 3. Critical Marangoni number $M_{c}$ for different values of $L$ and $R$ with $f(z)=1, N_{1}=0.5, N_{3}=2.0, N_{5}=1.0$.

\begin{tabular}{ccccc}
\hline & $\boldsymbol{L}=\mathbf{0}$ & $\boldsymbol{L}=\mathbf{1 0 0}$ & $\boldsymbol{L}=\mathbf{2 0 0}$ & $\boldsymbol{L}=\mathbf{5 0 0}$ \\
\hline $\boldsymbol{R}$ & $\boldsymbol{M}_{\boldsymbol{c}}$ & $\boldsymbol{M}_{\boldsymbol{c}}$ & $\boldsymbol{M}_{\boldsymbol{c}}$ & $\boldsymbol{M}_{\boldsymbol{c}}$ \\
\hline 0 & 98.5737 & 94.4581 & 90.3407 & 77.9774 \\
100 & 94.9229 & 90.8073 & 86.6899 & 74.3266 \\
200 & 91.2721 & 87.1565 & 83.0392 & 70.6758 \\
1000 & 62.0657 & 57.9502 & 53.8328 & 41.4694 \\
2135.89 & 20.5967 & 16.4812 & 12.3638 & 0.000432 \\
2474.48 & 8.2355 & 4.11997 & 0.002584 & \\
2587.33 & 4.11558 & 0.00004762 & & \\
2700 & 0.002234 & & & \\
\hline
\end{tabular}

Table 4. The values of critical wave number $a_{c}^{2}$ for various values of $N_{1}, N_{3}, N_{5}$ and $L$ with $f(z)=1$ and for all values of $\boldsymbol{R}$.

\begin{tabular}{cccccccc}
\hline \multicolumn{4}{c}{$L=\mathbf{1 0 0}, \boldsymbol{R}=\mathbf{1 0 0}$} & \multicolumn{5}{c}{$L=\mathbf{2 0 0}, \boldsymbol{R}=\mathbf{1 0 0}$} \\
\hline$N_{1}$ & $N_{3}$ & $N_{5}$ & $a_{c}^{2}$ & $N_{1}$ & $N_{3}$ & $N_{5}$ & $a_{c}^{2}$ \\
\hline 0.1 & 2 & 1 & 5.85319 & 0.1 & 2 & 1 & 5.7939 \\
0.5 & & & 5.85469 & 0.5 & & & 5.81864 \\
1 & & & 5.75689 & 1 & & & 5.73666 \\
0.1 & 2 & 1 & 5.85319 & 0.1 & 2 & 1 & 5.7939 \\
& 4 & & 5.85167 & & 4 & & 5.7939 \\
& 6 & & 5.85103 & & 6 & & 5.7939 \\
0.1 & 2 & 0.5 & 5.85319 & 0.1 & 2 & 0.5 & 5.7939 \\
& & 1 & 5.85319 & & & 1 & 5.7939 \\
& & 1.5 & 5.85319 & & & 1.5 & 5.7939 \\
\hline
\end{tabular}

conditions for an appropriate basic temperature gradient, we can also make an appropriate decision delaying the convection. It has been observed that parabolic temperature profile is the most stabilizing temperature profile and step function is the most destabilizing temperature profile.

\section{Acknowledgements}

The authors would like to thank their respective managements for the support in completing this work. One of the authors (TVJ) would like to acknowledge the support of the Christ University administration on deputing him to the $\mathrm{Ph} . \mathrm{D}$ program.

\section{REFERENCES}

[1] J. R. A. Pearson, “On Convection Cells Induced by Sur- face Tension," Journal of Fluid Mechanics, Vol. 4, No. 5, 1958, pp. 489-500. doi:10.1017/S0022112058000616

[2] D. A. Nield, "Surface Tension and Buoyancy Effects in Cellular Convection," ZAMM, Vol. 17, No. 1, 1966, pp. 131-139. doi:10.1007/BF01594092

[3] N. Rudraiah, V. Ramachandramurthy and O. P. Chandana, "Surface Tension Driven Convection Subjected to Rotation and Non-Uniform Temperature Gradient," International Journal of Heat and Mass Transfer, Vol. 28, No. 8, 1985, pp. 1621-1624. doi:10.1016/0017-9310(85)90264-9

[4] T. Maekawa and I. Tanasawa, "Effect of Magnetic Field and Buoyancy on Onset of Marangoni Convection," International Journal of Heat and Mass Transfer, Vol. 31, No. 2, 1988, pp. 285-293. doi:10.1016/0017-9310(88)90011-7

[5] G. S. R. Sarma, "Marangoni Convection in a Fluid Layer under the Action of a Transverse Magnetic Field," Space Research, Vol. 19, 1979, pp. 575-578.

[6] G. S. R. Sarma, "Marangoni Convection in a Liquid Layer under the Simultaneous Action of a Transverse Magnetic Field and Rotation," Advances in Space Research, Vol. 1, No. 5, 1981, pp. 55-58. doi:10.1016/0273-1177(81)90151-4

[7] M. Takashima, "Surface Tension Driven Instability in a Horizontal Liquid Layer with a Deformable Free Surface Part I Steady Convection," Journal of the Physical Society of Japan, Vol. 50, No. 8, 1981, pp. 2745-2750. doi:10.1143/JPSJ.50.2745

[8] S. K. Wilson, "The Effect of a Uniform Magnetic Field on the Onset of Steady Bénard-Marangoni Convection in a Layer of Conducting Fluid," Journal of Engineering Mathematics, Vol. 27, No. 2, 1993, pp. 161-188. doi:10.1007/BF00127480

[9] S. K. Wilson, "The Effect of a Uniform Magnetic Field on the Onset of Steady Marangoni Convection in a Layer of Conducting Fluid with a Prescribed Heat Flux at Its Lower Boundary," Physics of Fluids, Vol. 6, No. 11, 1994, pp. 3591-3560. doi:10.1063/1.868417

[10] I. Hashim and S. K. Wilson, "The Onset of Bénard-Marangoni Convection in a Horizontal Layer of Fluid," International Journal of Engineering Science, Vol. 37, No. 5, 1999, pp. 643-662. doi:10.1016/S0020-7225(98)00084-6

[11] R. J. Turnbull, "Electro Convective Instability with a Stabilizing Temperature Gradient. I. Theory," Physics of Fluids, Vol. 11, No. 12, 1968, pp. 2588-2596. doi:10.1063/1.1691864

[12] R. J. Turnbull and R. J. Melcher, "Electrohydrodynamic Rayleigh-Taylor Bulk Instability," Physics of Fluids, Vol. 12, No. 6, 1969, pp. 1160-1166. doi:10.1063/1.1692646

[13] M. Takashima and K. D. Aldridge, "The Stability of a Horizontal Layer of Dielectric Fluid under the Simultaneous Action of a Vertical dc Electric Field and a Vertical Temperature Gradient," The Quarterly Journal of Mechanics and Applied Mathematics, Vol. 29, No. 1, 1976, pp. 71-87.

[14] P. J. Stiles, "Electro Thermal Convection in Dielectric Liquids," Chemical Physics Letters, Vol. 179, No. 3, 1991, 


\section{pp. 311-315. doi:10.1016/0009-2614(91)87043-B}

[15] P. J. Stiles, F. Lin and P. J. Blennerhassett, "Convective Heat Transfer through Polarized Dielectric Liquids," Physics Fluids, Vol. 5, No. 12, 1993, pp. 3273-3279. doi:10.1063/1.858684

[16] P. G. Siddeshwar, "Oscillatory Convection in Viscoelastic, Ferromagnetic/Dielectric Liquids," International Journal of Modern Physics B, Vol. 16, No. 17-18, 2002, pp. 2629-2635. doi:10.1142/S0217979202012761

[17] P. G. Siddeshwar and A. Abraham, "Effect of Time-Periodic Boundary Temperatures/Body Force on RayleighBénard Convection in a Ferromagnetic Fluid," Acta Mechanica, Vol. 161, No. 3-4, 2003, pp. 131-150.

[18] P. G. Siddeshwar and A. Abraham, "Rayleigh-Bénard Convection in a Dielectric Liquid: Imposed Time-Periodic Boundarytemperatures," Chamchuri Journal of Mathematics, Vol. 1, No. 2, 2009, pp. 105-121.

[19] P. G. Siddeshwar and A. T. Y. Chan, "Ferrohydrodynamic and Electrohydrodynamics Instability in Viscoelastic Liquids: An Analogy," Proceedings 4th International Conference on Fluid Mechanics, Dalian, 20-30 July 2004, pp. 167-172.

[20] I. S. Shivakumara, M. S. Nagashree and K. Hemalatha, "Electrothermoconvective Instability in a Heat Generating Dielectric Fluid Layer," International Communications in Heat and Mass Transfer, Vol. 34, No. 9-10, 2007, pp. 1041-1047. doi:10.1016/j.icheatmasstransfer.2007.05.006

[21] N. Rudraiah, B. M. Shankar and C.-O. Ng, "Electrohydrodynamic Stability of Couple Stress Fluid Flow in a Channel Occupied by a Porous Medium," Special Topics \& Reviews in Porous Media-An International Journal, Vol. 2, No. 1, 2011, pp. 11-22.

[22] P. G. Siddeshwar and D. Radhakrishna, "Linear and Nonlinear Electroconvection under AC Electric Field,"
Communications in Nonlinear Science and Numerical Simulation, Vol. 17, No. 7, 2012, pp. 2883-2895. doi:10.1016/j.cnsns.2011.11.009

[23] A. C. Eringen, "Theory of Micropolar Fluids," International Journal of Engineering Science, Vol. 16, No. 1, 1966, p. 1.

[24] B. Datta and V. U. K. Sastry, "Thermal Instability of a Horizontal Layer of Micropolar Fluid Heated from Below," International Journal of Engineering Science, Vol. 14, No. 7, 1976, pp. 631-637. doi:10.1016/0020-7225(76)90005-7

[25] P. G. Siddheshwar and S. Pranesh, "Magnetoconvection in a Micropolar Fluid," International Journal of Engineering Science, USA, Vol. 36, No. 10, 1998, pp. 1173 1181. doi:10.1016/S0020-7225(98)00013-5

[26] P. G. Siddheshwar and S. Pranesh, "Magnetoconvection in Fluids with Suspended Particles under $1 \mathrm{~g}$ and ug," International Journal of Aerospace Science and Technology, France, Vol. 6, No. 2, 2001, pp. 105-114. doi:10.1016/S1270-9638(01)01144-0

[27] P. G. Siddheshwar and S. Pranesh, "Suction-Injection Effects on the Onset of Rayleigh-Bénard-Marangoni Convection in a Fluid with Suspended Particles," Acta Mechanica, Germany, Vol. 152, No. 1-4, 2001, pp. 241-252. doi:10.1007/BF01176958

[28] S. Pranesh and R. Baby, "Effect of Non-Uniform Temperature Gradient on the Onset of Rayleigh-Bénard Electro Convection in a Micropolar Fluid," Applied Mathematics, Vol. 3, No. 5, 2012, pp. 442-450. doi:10.4236/am.2012.35067

[29] S. Pranesh and A. Kumar, "Effect of Non-Uniform Basic Concentration Gradient on the Onset of Double-Diffusive Convection in Micropolar Fluid," Applied Mathematics, Vol. 3, No. 5, 2012, pp. 417-424. doi:10.4236/am.2012.35064 